\title{
Dedicated controller design for a dual-stage opto-mechatronic system
}

\author{
M. Stalder, Y. Michellod, Ph. Mullhaupt and D. Gillet \\ École Polytechnique Fédérale de Lausanne, Switzerland
}

\begin{abstract}
To improve the resolution of the Very Large Telescope Interferometer (VLTI) a two-stage mechanical system, a so called Differential Delay Line (DDL), is developed jointly by the EPFL and the Observatory of Geneva. The system is designed to reach nanometer accuracy at high bandwidth over large displacements. The coarse stage features a permanent magnet (PM) stepper motor driving a lead screw connected to a double-parallelogram flexure with notch-hinges (blade) guiding system, and the fine stage features a stacked piezoelectric device, combine to one single measurable output.

This paper compares different control approaches for the DDL with their respective advantages and disadvantages. The developed control methods are based on modern linear and nonlinear control theory. The performance of the control schemes is illustrated via simulation and measurement on the available prototype. The new developed methods are compared to the currently implemented decoupled SISO design which features a direct-coil controller for the coarse stage and a simple PID-controller for the fine one.
\end{abstract}

\section{INTRODUCTION}

Earthbound astrometry is limited by atmospheric turbulence, the reason for which extra-solar planets and other faint objects are currently out of reach. Therefore, the European Southern Observatory (ESO) is developing the phase-referenced imaging and micro-arcsecond astrometry (PRIMA) facility for the VLTI to solve this problem. To enable full performance of PRIMA the Differential Delay Line (DDL) is needed. It dynamically compensates for the differential Optical Path Difference $(\Delta \mathrm{OPD})$ to reduce the effect of atmospheric disturbance and give stabilized interference fringes for phase-referenced imaging [4], [5], [7], [11].

The proposed design of the DDL corresponds to a dualstage double-input single-output (DISO) system, which is also seen in hard-disk drives (HDD). Presently, a decoupled SISO control scheme [13] is implemented on the DDL prototype. Without changing this structure, two knew approaches for the coarse-stage control are introduced, one using a flatness based controller [8], [6], [3] and the other a classical PI controller. Furthermore, two global control structures will be designed. One of them uses the PQ method dedicated to dual-stage systems and mostly used in HDD control [12], [14]. Furthermore, an optimal linear quadratic regulator [10], [15], which controls both stages, is proposed. All the new methods benefit from an exact feedback linearization technique for the PM stepper motor similar to the Park transformation [2], [1], [17].

The paper is organized as follows. In section II, a dynamical model of the DDL is developed. Section III introduces the different control laws to be applied to the DDL, followed by some simulation and experimental results in section IV.

\section{MODELING}

The model of the DDL is divided into: i) the coarse stage, formed by the PM stepper motor, ii) the lead screw and iii) the blade guiding system (a double-parallelogram flexure with notch hinges), and iv) the fine stage, featuring the piezoelectric actuator.

\section{A. Coarse stage}

Mainly due to energy consumption issues, the project hardware was modified from voltage control, as presented in [13], to current control. The standard model for a PM stepper motor can thus be reduced to:

$$
\begin{aligned}
\frac{d \theta}{d t}= & \omega \\
\frac{d \omega}{d t}= & -\frac{K_{m}}{J} i_{a} \sin (N \theta)+\frac{K_{m}}{J} i_{b} \cos (N \theta) \\
& -\frac{F_{f}}{J} \omega-\frac{T_{L}}{J} \\
y_{c}= & \gamma \theta
\end{aligned}
$$

Where $i_{a}$ and $i_{b}$ are the two inputs applied on phases $a$ and $b$ of the motor; $K_{m}$ is the electromagnetic coefficient; $N$ the number of steps per revolution per phase; $J$ the inertial load relative to the rotation axis; $F_{f}$ the viscous friction and $T_{L}$ the external torque load. $y_{c}$ is the linear position of the coarse stage (the origin being the position where no force is applied to the blades) related by $\gamma$ to the corresponding angle $\theta$.

The blade guiding system induces a reaction force on the coarse stage actuator, which varies drastically with the position (the force varies from $-150 \mathrm{~N}$ to $150 \mathrm{~N}$ ). The force-deformation characteristic can be modeled with a thirdorder polynomial [9, page 150]. The torque-deformation characteristic is assumed to be of the same form, thus the external torque is modeled as

$$
T_{L}=\left(k_{1}+k_{2} \theta^{2}\right) \theta .
$$

The effects of the lead screw are included in the parameters of the PM stepper motor model and the blade-guidingsystem model.

1) Parameter identification: Parameter identification was undertaken in two steps, so as to capture the fast dynamics of the motor as well as the effects of the blade guiding system. The final parameters are presented in table I. The identification process showed that $k_{2}=0$ and therefore a linear model for the external torque load is sufficient:

$$
T_{L}=k_{1} \theta \text {. }
$$


TABLE I

IDENTIFIED COARSE STAGE PARAMETERS

\begin{tabular}{|l||c|c|c|c|c|}
\hline Parameter & $K_{m}$ & $J$ & $F_{f}$ & $k_{1}$ & $k_{2}$ \\
\hline Value & 1.1282 & $2 \mathrm{e}-4$ & 0.1220 & 0.03 & 0 \\
\hline
\end{tabular}

2) Linearization: The PM stepper-motor model for voltage control is often linearized by the Park-transformation [2], [1], [17]. For the new model (1), this transformation can be simplified through setting $i_{d}=0$, so as to get the linearizing inputs:

$$
\begin{aligned}
i_{a} & =-\sin (N \theta) I \\
i_{b} & =\cos (N \theta) I
\end{aligned}
$$

With the new input $I$, model (1) is linearized to

$$
\begin{aligned}
\frac{d \theta}{d t} & =\omega \\
\frac{d \omega}{d t} & =-\frac{F_{f}}{J} \omega-\frac{k_{1}}{J} \theta+\frac{K_{m}}{J} I
\end{aligned}
$$

\section{B. Fine stage}

The piezoelectric actuator available is a tripod design of three parallel-mounted piezo-stack actuators. Drift and hysteresis can be compensated in two ways:

1) by internal electronics, provided by "Physik Instrumente" (PI), so that the input-output behavior is almost linear.

2) by adding a feed-forward loop containing the inverse model of the hysteresis [16].

Currently, the first of the above methods is used, which makes black-box identification very convenient. For this purpose, a pseudo-random binary signal (PRBS) was applied to the system and the data were collected with a sampling frequency of $5000 \mathrm{~Hz}$. An ARX model with coefficients $n_{a}=3, n_{b}=2$ and $n_{k}=1$ gave a reasonable simple and good model. The ARX model obtained is given by

$$
\begin{aligned}
A(q) y(t) & =B(q) u\left(t-n_{k}\right)+e(t) \\
A(q) & =1+a_{1} q^{-1}+\ldots+a_{n_{a}} q^{-n_{a}} \\
B(q) & =b_{1} q^{-1}+\ldots+b_{n_{b}} q^{-n_{b}+1}
\end{aligned}
$$

The identified parameters can be found in table II.

\section{CONTROL STRATEGIES}

\section{A. Decoupled SISO design}

The overall structure described in Fig. 1 is equivalent to the one presented in [13], as well as the specific fine-stage controller. For the coarse-stage control several methods are designed, which are presented in the following sections.

TABLE II

IDENTIFIED FINE STAGE PARAMETERS

\begin{tabular}{|l||c|c|c|c|c|}
\hline Parameter & $a_{1}$ & $a_{2}$ & $a_{3}$ & $b_{1}$ & $b_{2}$ \\
\hline Value & -1.715 & 0.9232 & -0.1405 & -0.002362 & 0.2073 \\
\hline
\end{tabular}

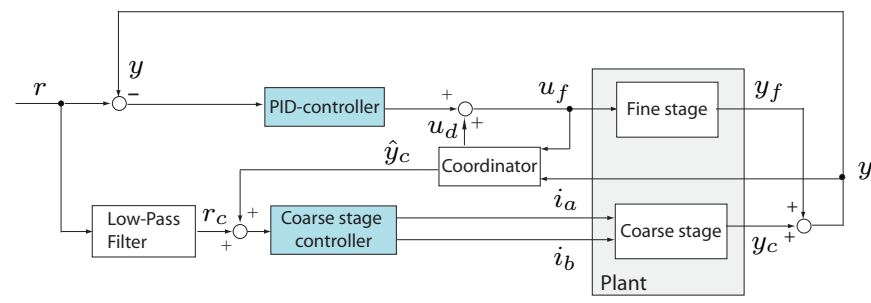

Fig. 1. Decoupled SISO design with undefined coarse stage controller

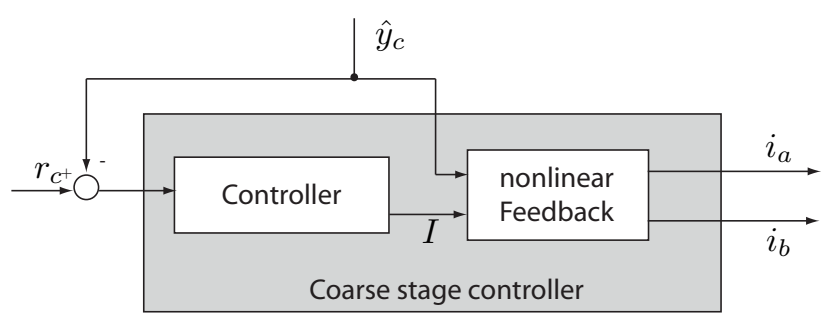

Fig. 2. Coarse stage controller based on feedback linearization

1) Direct sine/cosine coarse-stage controller: The directcoil controller proposed in [13] also applies to current control. Consequently, the following controller is proposed:

$$
\begin{aligned}
i_{a} & =I_{\text {peak }} \sin (\alpha) \\
i_{b} & =I_{\text {peak }} \cos (\alpha) \\
e_{c} & =r_{c}-\hat{y}_{c} \\
\alpha & =\frac{1}{T_{i}} \int_{0}^{t} e_{c}(t) \mathrm{d} t,
\end{aligned}
$$

where $\alpha$ is the phase of the stator magnetic field; $I_{\text {peak }}$ is the peak current applied to the coils of the motor; $e_{c}$ is the coarse-stage tracking error, i.e the difference between the estimated position of the PM stepper motor $\hat{y}_{c}$ and the low-pass filtered reference $r_{c}$. To avoid de-sychronisation between rotor and the generated magnetic field, the variation of the controlled angle $\alpha$ has to be limited [13].

Since the peak current is constant, this particular controller dissipates power constantly, even if the PM stepper motor is at rest. Deriving the RMS power dissipation leads to following relation:

$$
P_{R M S}=2 R I_{\text {peak }}^{2}
$$

2) Coarse-stage controller based on feedback linearization: To avoid the constant power dissipation a new coarse stage controller has to be designed. Using the concept of exact feedback linearization the coarse stage controller can be divided into a control part and a part reconstructing the inputs $i_{a}$ and $i_{b}$ (Fig. 2). From a theoretical point of view, the "coarse stage"-block (Fig. 1) and the "nonlinear feedback"block (Fig. 2) build exactly the linearized motor model (5).

a) PI-linearized: A classic PI-controller with the following transfer function is the first choice:

$$
P I(s)=K_{p} \cdot\left(1+\frac{1}{T_{i} s}\right)
$$


An integrator anti-reset windup (ARW) is introduced, of the simple form:

$$
\begin{aligned}
& u_{i_{k}}=0 \quad \text { if } e_{k}>\epsilon \\
& u_{i_{k}}=u_{i_{k-1}}+\frac{h}{T_{i}} e_{k} \text { if } e_{k} \leq \epsilon
\end{aligned}
$$

b) Flatness based control: The flatness property of a system is characterized by the existence of a flat output, which parameterizes the inputs and the system states with a finite number of its derivatives. By planning the trajectories of the flat output, one gets the corresponding system states and inputs without integrating a differential equation. The parameterized input linearizes the system and thus the flatness property is similar to dynamic feedback linearization, therefore the term exact feedforward linearization based on differential flatness is also used [6], [8], [3].

Since the coarse-stage model is considered in its linearized form (5), the simplest flat output to the system is

$$
y_{f}=\gamma \theta
$$

From the desired trajectories $y_{f}^{*}, \dot{y}_{f}^{*}$ and $\ddot{y}_{f}^{*}$ the nominal input $I^{*}$ to the system (5) is constructed:

$$
I^{*}=\frac{1}{\gamma K_{m}}\left[J \ddot{y}_{f}^{*}+F_{f} \dot{y}_{f}^{*}+k_{1} \frac{y_{f}^{*}}{\gamma}\right]
$$

To stabilize the system around the trajectory the new input $v$ is designed as

$$
v=\ddot{y}_{f}^{*}-2 k\left(\dot{y}_{f}-\dot{y}_{f}^{*}\right)-k^{2}\left(y_{f}-y_{f}^{*}\right),
$$

where $k>0$ is a design parameter. Combining (15) and (16) gives the control law

$$
I=\frac{1}{\gamma K_{m}}\left[J\left(\ddot{y}_{f}^{*}-k^{2} e-2 k \dot{e}\right)+F_{f} \dot{y}_{f}^{*}+k_{1} \frac{y_{f}^{*}}{\gamma}\right],
$$

with $e=y_{f}-y_{f}^{*}$ and $\dot{e}=\dot{y}_{f}-\dot{y}_{f}^{*}$.

\section{B. $P Q$ controller}

The PQ method is dedicated to dual-stage DISO systems, which is mostly used for the control of HDDs. The method reduces the DISO design problem into two SISO design problems [12], [14].

To apply the PQ method, linear models of the fine stage and coarse stage are needed. Converting the identified finestage ARX model into a transfer function yields the following function

$$
G_{1}=\frac{b_{2} s^{2}+b_{1} s+b_{0}}{s^{3}+a_{2} s^{2}+a_{1} s+a_{0}},
$$

where the transfer function coefficients are given in table III. The linearized coarse-stage model (5) can easily be written

TABLE III

FINE STAGE CONTINUOUS-TIME TRANSFER FUNCTION COEFFICIENTS

\begin{tabular}{|l||c|c|c|}
\hline Nominator & $b_{0}$ & $b_{1}$ & $b_{2}$ \\
\hline Value & $6.3 \cdot 10^{10}$ & $-2.2 \cdot 10^{6}$ & -423.2 \\
\hline Denominator & $a_{0}$ & $a_{1}$ & $a_{2}$ \\
\hline Value & $2.1 \cdot 10^{10}$ & $2.3 \cdot 10^{7}$ & 9814 \\
\hline
\end{tabular}

as a transfer function:

$$
G_{2}=\frac{K_{m} \gamma}{J s^{2}+F_{f} s+k_{1}}
$$

The numerical values for $K_{m}, J, F_{f}$ and $k_{1}$ are given in Table I.

1) Actuator-output allocation: As proposed in [13], the stepper motor's contribution should be limited, so that it keeps the piezo actuator within saturation range. Instead of filtering the reference for the coarse stage, the PQ method allows to choose the relative actuator-output allocation by setting the $0-\mathrm{dB}$ crossover frequency of the $P Q$ transfer function, where $P=\frac{G_{2}}{G_{1}}$ and $Q=\frac{C_{2}}{C_{1}}$ [12], [14]. Typically, the $0-\mathrm{dB}$ crossover frequency is chosen at around $10 \mathrm{~Hz}$. Moreover, an integrator for each actuator is added so as to eliminate the residual steady-state errors.

The uncompensated system $P$ in Fig. 3 has a phase margin of $88^{\circ}$ at $0-\mathrm{dB}$ crossover frequency $115 \mathrm{~Hz}$. Because the relative-output allocation should take place at around $10 \mathrm{~Hz}$, the $0-\mathrm{dB}$ crossover frequency needs to be lowered, which is achieved with a small static gain. So as to have integrators on both actuators, the following $Q$ is proposed:

$$
Q=\frac{T_{i 1} s}{T_{i 2} s} \text {. }
$$

This induces a slow integrator on the coarse stage and a fast one on the fine stage. The static gain compensation is equivalent to $\frac{T_{i 1}}{T_{i 2}}$. In Fig. 3, one can see that the compensated system $P Q$ has a phase margin of $89^{\circ}$ at $0-\mathrm{dB}$ crossover $8.2 \mathrm{~Hz}$, where the phase margin fulfills the requirement for constructive interference [12], [14]. With this choice of $Q$, the $P Q$ system is not stable since the identified fine-stage model $G_{1}$ happens to be non-minimum phase.

The simplest $C_{1}$, so that $C_{2}$ is proper, is

$$
C_{1}=\frac{1}{T_{i 1} s}
$$

$C_{2}$ then becomes

$$
C_{2}=\frac{1}{T_{i 2} s}
$$

which is realizable.

2) Loop shaping for overall performance: Designing $C_{0}$, the overall performance of the system can be improved. The closed-loop bandwidth of $G_{\text {siso }}=C_{1} G_{1}+C_{2} G_{2}$ (Fig. 4) needs to be increased to achieve the demanded performance. A simple static gain is not enough, because the new phase margin is negative. Therefore, an additional lead compensator is incorporated, which contributes to the missing phase so as to stabilize the loop. The following transfer function for $C_{0}$ arises:

$$
C_{0}=K_{p}\left(\frac{s+\frac{1}{T_{2}}}{s+\frac{1}{\beta_{2} T_{2}}}\right)
$$




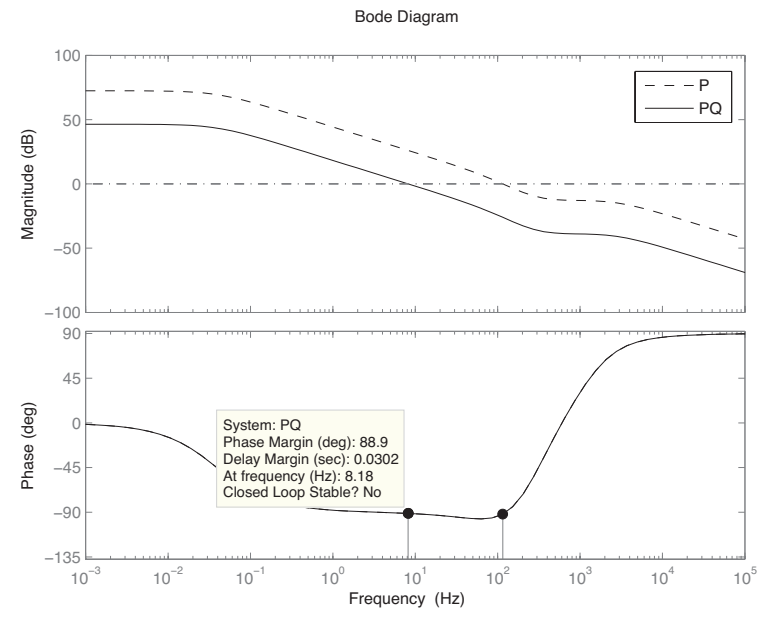

Fig. 3. Influence of the $\mathrm{Q}$ transfer function

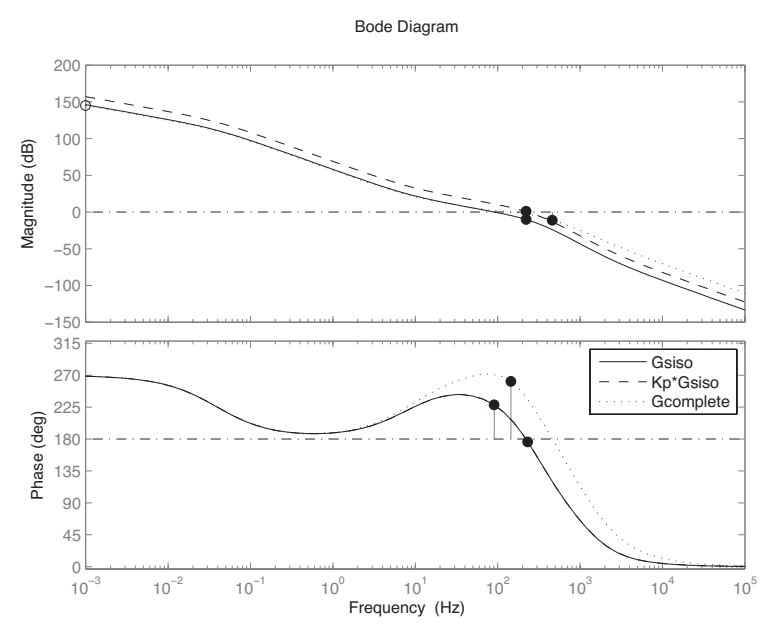

Fig. 4. Frequency response of $G_{\text {siso }}, K_{P} \cdot G_{\text {siso }}$ and $C_{0} \cdot G_{\text {siso }}$

3) Overall control structure: The controller designed in sections III-B. 1 and III-B.2 is based on a linear model of the plant. As seen in section II-A the coarse-stage model has highly non-linear dynamics, hence its linearization incorporates the variable transformation (4). In practice this linearization is inverted through reconstructing the inputs $i_{a}$ and $i_{b}$ with the relationship (4). To do so, an observer is needed to provide an estimate of the coarse-stage position. It is sufficient to use the static observer proposed in [13]:

$$
\begin{aligned}
y & =y_{f}+y_{c} \\
\hat{y}_{c} & =y-P_{D C} \cdot \operatorname{Sat}\left[u_{f}\right],
\end{aligned}
$$

where $P_{D C}$ is a static gain model of the piezo. Thus, the PQ controller is implemented based on the control structure appearing in Fig. 5.

\section{LQ controller}

To guarantee tracking, the reference is fed to the plant inputs through the block " $\mathrm{N}$ " in Fig. 6. Considering the benefits of the dual-stage design, the reference is split by tracking the actual reference $r$ with the coarse stage and

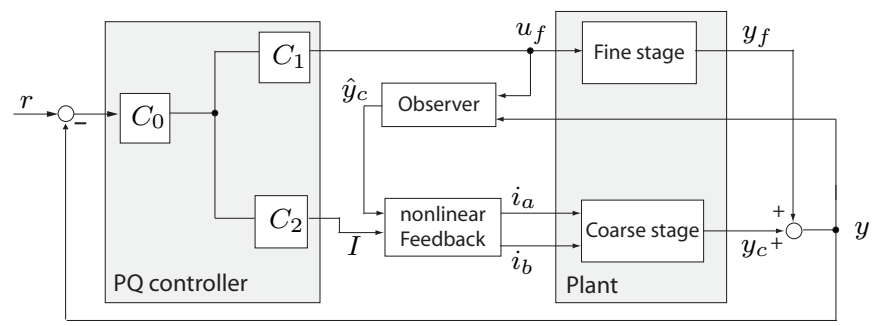

Fig. 5. PQ control structure for the DDL

the error between the reference $r$ and the estimated coarsestage position $\hat{y}_{c}$ with the fine stage, where the coarse stage estimate is provided as in (24). So as to reject perturbations and model errors, an integrator term on the error is added to the fine-stage reference. Hence, the equations of $N$ :

$$
\begin{aligned}
u_{c}^{*} & =r \cdot\left(K_{c}\right)^{-1} \\
u_{f}^{*} & =\left(r-\hat{y}_{c}+i_{e}\right) \cdot\left(K_{f}\right)^{-1} \\
i_{e} & =\int(r-y) \mathrm{d} t
\end{aligned}
$$

where $K_{c}$ and $K_{f}$ represent the closed-loop static gain of the coarse stage, resp. fine stage.

The weighting matrices of the linear quadratic regulator are chosen in a way to enforce the dual-stage nature of the system. This means that, on one hand, the coarse stage is tuned quite softly, so that it gently follows the reference, but, on the other hand, the fine stage is tuned very aggressively so as to give the necessary tracking precision.

In simulation, the classical Kalman estimator of the LQG controller is used. From an implementation point of view, a Kalman filter is not applicable, due to computational burden. Instead, the fine-stage states are estimated by simulating the model in parallel and the coarse-stage position is estimated using (24). The coarse-stage velocity is estimated through numerically differentiating the estimated coarse-stage position. In this case no LQ estimator is in the loop, therefore the design corresponds to a LQR controller.

Since the proposed exact feedback linearization (4) does not affect the system states, only the inputs $i_{a}$ and $i_{b}$ have to be reconstructed. Alltogether this gives the control structure in Fig. 6.

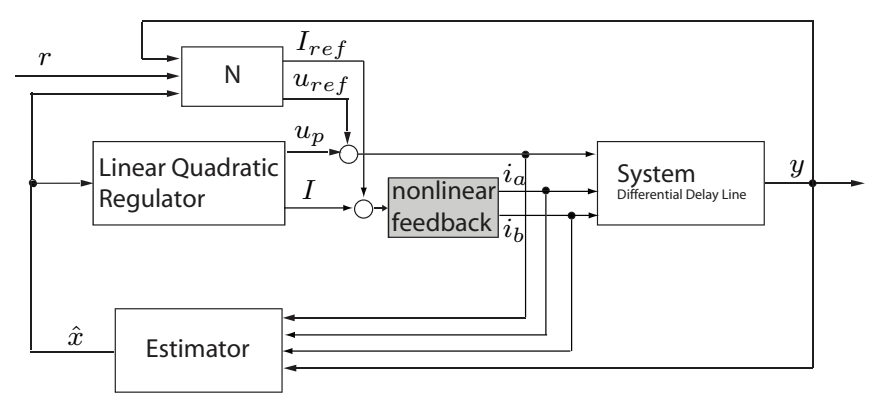

Fig. 6. LQG overall control structure 


\section{Overview}

There are five different methods to be compared by simulation and tested on the real system. Three are based on the decoupled SISO structure tagged; "direct coil", "PIlinearized" and "flatness based" (section III-A). Further two global structures, "PQ" (section III-B) and "LQG", resp "LQR" (section III-C), were designed. In the next sections, only one of these tags will be used to refer to one particular control law.

All methods, except the direct-coil controller, use the exact feedback linearization technique. The driving force, resp. the torque generation, of the methods using exact feedback linearization and the direct-coil control is fundamentally different. Linearizing the model (1) reduces it to a SISO system (5) with the single input $I$. Using this input to reconstruct $i_{a}$ and $i_{b}$, only the amplitude of the inputs can be influenced. The phase of the stator magnetic field is fixed at $90^{\circ}$ with respect to the actual rotor position $\theta$. In case of direct-coil control, the amplitude of the input currents is fixed to $I_{\text {peak }}$, but the phase of the stator magnetic field can be altered so as to generate the necessary torque.

\section{RESULTS}

In pure tracking mode, a realization of a stochastic Kolmogorov process (with a frequency content up to 250 $\mathrm{Hz}$ ) is used as a reference. This signal is representative of the atmospheric disturbances encountered. In reality, the reference is not a pure Kolmogorov signal. From time to time, there is a brusque change in position or even a switch in stroke from one end to the other. The behavior of the system in such cases can be tested through performing step responses with displacements of different amplitudes.

The controllers need to track the reference with less than $70 \mathrm{~nm}$ RMS error, in pure tracking, and cover the whole bandwidth of $250 \mathrm{~Hz}$. In addition, they need to be robust all over the stroke range and for all the different kind of references.

\section{A. Simulation results}

1) Kolmogorov tracking: To analyze the quality of the tracking performance, several characteristics are evaluated.

- The root mean square error

$$
\mathrm{RMSE}=\sqrt{\frac{1}{n} \sum_{i=1}^{n} e_{i}^{2}},
$$

where $n$ is the number of samples.

- The maximal absolute error

$$
|e|_{\max }=\max _{e}\left|e_{i}\right| .
$$

- The mean absolute error

$$
\bar{e}=\frac{1}{n} \sum_{i=1}^{n}\left|e_{i}\right|,
$$

where $n$ is the number of samples.

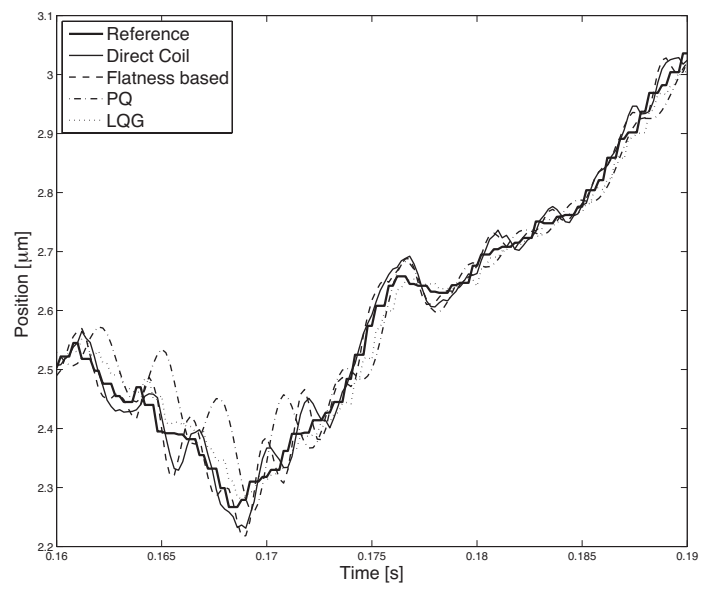

(a) Detail of the DDL output position

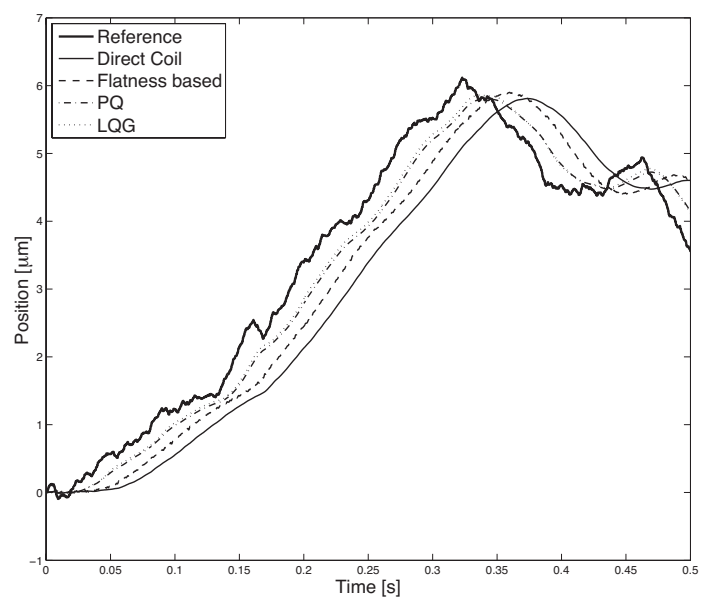

(b) Detail of the coarse stage position

Fig. 7. Kolmogorov tracking in simulation with different control methods

- The power dissipation

$$
\mathrm{P}_{R M S}=2 R\left(i_{a_{R M S}}^{2}+i_{b_{R M S}}^{2}\right),
$$

with $R=0.5 \Omega$.

Each controller is simulated over 2 seconds in tracking mode. In Fig. 7(a) a detail of the DDL output position is given and in Fig. 7(b) the corresponding coarse-stage position on a different time scale. One can see, in Fig. 7(a), that the PQ controller shows the largest deviations from the reference especially at the beginning, whereas in case of the LQG controller the DDL tracks very closely the reference. This is perfectly in line with the results in columns 1 to 3 in Table IV. All the different errors of the PQ controller are larger than for the LQG, with the other three controllers being somewhere in between. The direct coil, flatness based, and PI-linearized controller should have similar coarse-stage action, since their coarse-stage controller is given the same filtered reference. Fig. 7(b) shows that the direct-coil controller has a small delay with respect to the coarse-stage action under flatness-based control, which arises from the pure integration control (same effects as a low-pass filter) of the direct-coil controller (section III-A.1). The low-pass filter property of the integrator also has positive 
TABLE IV

TRACKING RESULTS IN SIMULATION

\begin{tabular}{|l|c|c|c|c|}
\hline & RMSE [nm] & $|e| \max [\mathrm{nm}]$ & $\bar{e}[\mathrm{~nm}]$ & $\mathrm{P}[\mathrm{W}]$ \\
\hline Direct Coil & 23 & 103 & 18 & 2.25 \\
\hline PI-linearized & 33 & 149 & 26 & $2.4 \mathrm{e}-5$ \\
\hline Flatness based & 27 & 118 & 21 & $4.5 \mathrm{e}-5$ \\
\hline PQ & 45 & 207 & 35 & $4.3 \mathrm{e}-5$ \\
\hline LQG & 15 & 84 & 20 & $4.5 \mathrm{e}-5$ \\
\hline
\end{tabular}

effects. The inaccurate coarse-stage position estimated does not affect the direct-coil controller, but induces a slight jitter of the coarse stage, which affects the tracking performance in terms of RMS, absolute and mean error (Table IV columns 1-3). Interesting to mention is that the coarse-stage action of the PQ and LQG controller are almost equal (Fig 7(b)), despite their different design approaches. Furthermore, the PQ controller shows worse performance for RMS, absolute and maximal error (columns 1-3 Table IV). This means that the optimal LQG controller treats the fine-stage action much better than the PQ controller.

As expected, all methods based on exact feedback linearization need much less energy as the direct-coil structure (Table IV column 4). However, the power dissipation is almost zero, which is not very realistic, but is explained by the absence of a detailed static-friction model.

\section{B. Experimental setup}

1) DDL prototype and laser metrology: The DDL prototype available at the EPFL is built from a PM stepper motor NEMA 23 from "Ultramotion", combined with a lead-screw which covers a full stroke of $70 \mathrm{~mm}$. The motor is powered by current a amplifier developed by the PRIMA consortium. Also, the blade guiding system is a tailor-made product. The piezoelectric S-325 is from PI with a course of $30 \mu \mathrm{m}$ and a bandwidth of approximately $300 \mathrm{~Hz}$.

The output is measured with an Agilent laser metrology.

2) Data acquisition hardware:

- VME rack: Two cards are installed into the VME rack to get access to the laser metrology: the Agilent 10897B laser board, which gives the position measured with subnanometer resolution, and the NI VME-MXI-2 board. The VME rack interfaces the memories of both boards, so that the measurements are available to a PC.

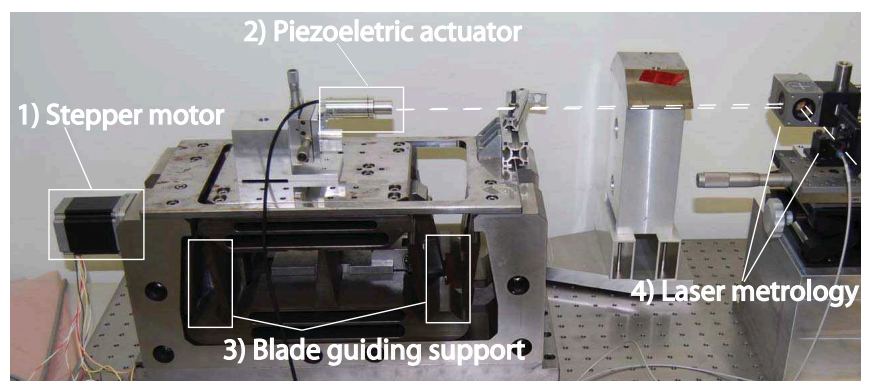

Fig. 8. Experimental setup: 1) Stepper motor, driving the coarse stage; 2) Piezoelectric actuator, driving the fine stage; 3) Double parallelogram flexure with notch-hinges; 4) Laser metrology with sub-nanometer resolution

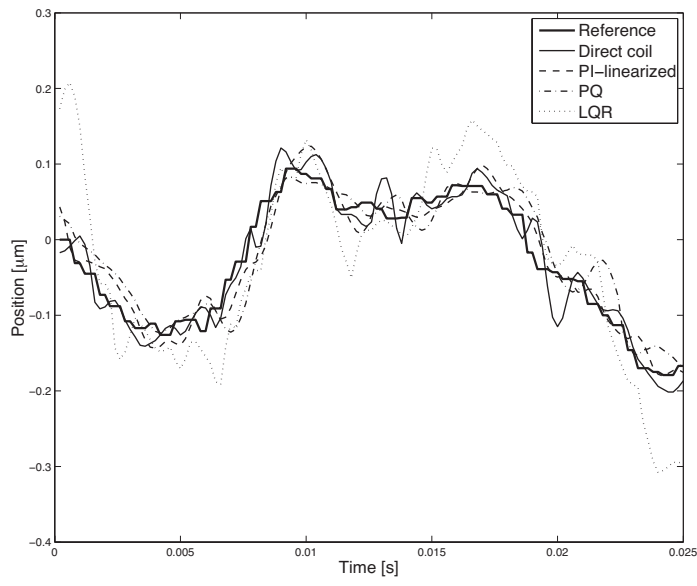

(a) Detail of DDL output position; time scale $0 \mathrm{~s}$ to $0.025 \mathrm{~s}$

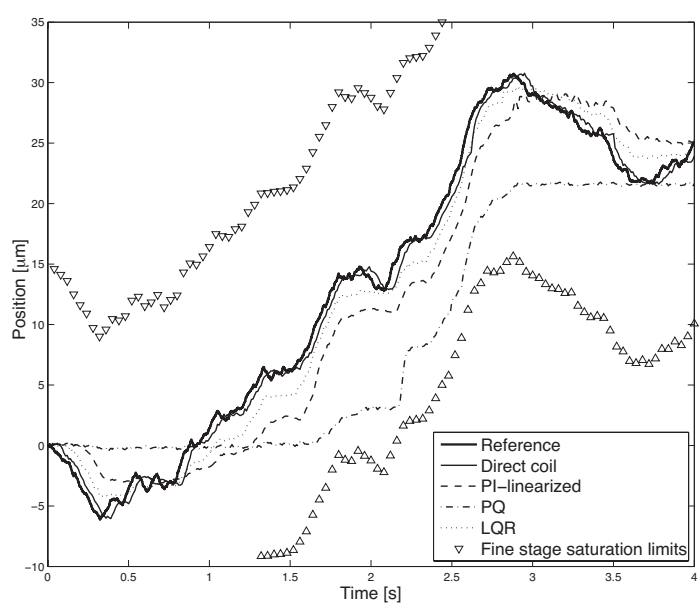

(b) Detail of the estimated coarse-stage position; time scale $0 \mathrm{~s}$ to $4 \mathrm{~s}$; the fine-stage saturation limits are marked by the triangles

Fig. 9. Kolmogorov signal tracking experience with different control laws

- NI PCI-MXI-2: This board is connected to the NI VME-MXI-2 board, so as to access the position measurement and to establish the real time control loop.

- NI PCI-6025E and NI PCI-6251: These cards provide the 3 analog outputs to control the piezoelectric actuator and the stepper motor. One of them is also used as timing source to synchronize the measurement and the excitation of the actuators.

3) Software: LabView 8.2 is used to implement the controllers. In a timed loop all the necessary operations are executed, where the loop is timed with the clock of one of the NI DAQ boards. To achieve good closed-loop performance, the sampling rate is selected to be $5 \mathrm{kHz}$.

In Fig. 8 a schematic representation of the complete installation is given.

\section{Experimentation results}

1) Kolmogorov tracking: Kolmogorov-signal tracking was executed over 10 seconds. In figure 9 , only a detail is given in order to make differences visible. The tracking performance is evaluated with the same relationships as in section IV-A.1. 
In Fig. 9(b) one can see that all the controllers guarantee that the coarse stage keeps the fine one within its saturation limits, which is necessary for the functionality of the system. In case of the PQ controller the coarse-stage action is quite at the limit of what is allowed (Fig. 9(b)); nevertheless the performance is similar to the one of the LQR controller in terms of RMS and mean error (Table V column 1 and 3). Furthermore, the three controllers based on the decoupled SISO design (direct coil, flatness based and PI-linearized) have similar RMS and mean errors (Table V column 1 and 3 ). Since they all use the same fine-stage controller, which gives the precision to the system, this is not surprising. In Fig. 9(a) the LQR shows a sort of oscillatory behavior and bigger deviation from the reference than the rest of the controllers. Checking the maximal error of the controllers (Table V column 2), one can see that just the LQR controller has the largest maximal error. Whereas for the direct coil and PQ controller the deviation peaks are less marked (Fig. 9(a)), also the maximal error of these controllers is smaller than for the LQR (Table V column 2). The PI-linearized controller does not show any oscillations around the reference (Fig. 9(a)), which is also confirmed by the smallest maximal error of all controllers (Table V column 2). In case of the PQ controller, one can also clearly see the effects of static friction on the coarse stage action (Fig. 9(b)), which leads to a sort of stepping behavior. The other controllers show a smoother coarse-stage movement especially the direct-coil controller (Fig. 9(b)). This controller also injects constantly a lot of energy into the system (section III-A) and thus the static friction does not affect the coarse-stage movement. Column 4 in Table V shows that all the controllers based on exact feedback linearization dissipate much less power in tracking than the direct-coil controller.

Based on the tracking performance in Table V the PIlinearized controller is rated best, since it has lowest values in RMS, absolute and maximal error and additionally the power dissipation is much less than for the direct-coil controller.

2) $5000 \mu \mathrm{m}$ step response: To test the system for sudden large displacements in the reference, step displacements of $5000 \mu \mathrm{m}$ are applied to the DDL. One experience is done in the center of the stroke, where no force is applied to the blades (Fig. 10(a)), and another towards the end of the stroke, where a big reaction force acts on the coarse-stage actuator (Fig. 10(b)).

In order to obtain the results illustrated in Fig. 10, strict rate limitations for speed have been introduced, for the controllers based on the decoupled SISO structure, as mentioned in [13]. With these measurements, taken the system with

TABLE V

TRACKING RESULTS IN IMPLEMENTATION

\begin{tabular}{|l|c|c|c|c|}
\hline & RMSE [nm] & $|e| \max [\mathrm{nm}]$ & $\bar{e}[\mathrm{~nm}]$ & $\mathrm{P}[\mathrm{W}]$ \\
\hline Direct Coil & 25 & 308 & 19.6 & 2.25 \\
\hline Flatness based & 26.6 & 147.5 & 20.7 & 0.016 \\
\hline PI-linearized & 22.8 & 133 & 17.7 & 0.017 \\
\hline PQ & 40 & 270 & 31 & 0.012 \\
\hline LQR & 44.8 & 640 & 30.7 & 0.015 \\
\hline
\end{tabular}

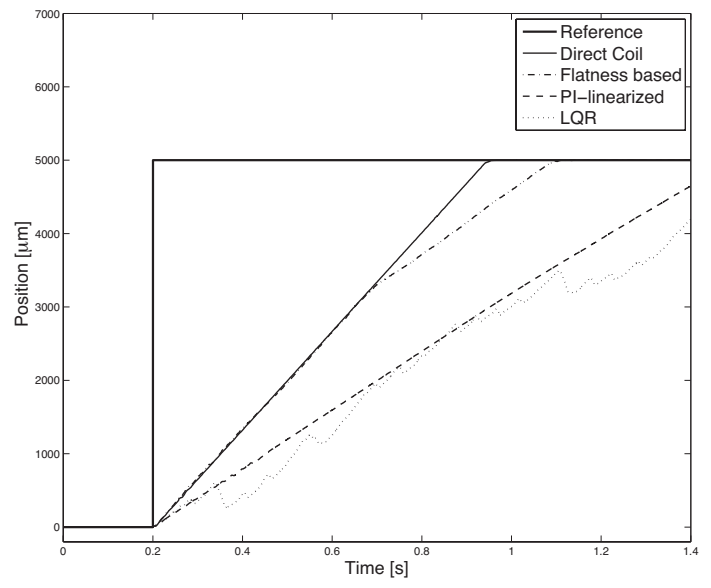

(a) DDL output position for a $5000 \mu \mathrm{m}$ step displacement at the center

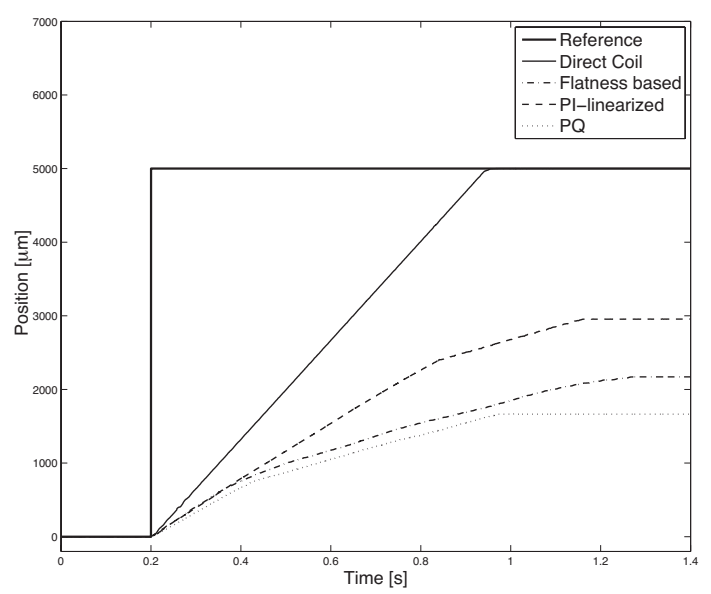

(b) DDL output position for a $5000 \mu \mathrm{m}$ step displacement towards the extremity of the stroke

Fig. 10. Tests of sudden large displacements in the tracking reference

direct-coil control performed best, since it allows the highest speed without destabilizing the system (Fig. 10(a)). In case of the LQR controller, no such speed limitation can be introduced, which leeds to a random behavior caused by desynchronization between rotor and the generated magnetic field (Fig. 10(a)). Similar behavior was also observed for the other methods based on exact feedback linearization depending on position and moving direction of the coarse stage. Towards the extremities of the stroke they also have problems to follow the reference. As one can see in Fig. 10(b), if the system is driven by a controller based on linearization, the DDL suddenly stops to move. The controllers do not allow the motor to generate enough torque to make the table move. With the direct coil controller, the system has no problems to follow the reference (Fig. 10(a)), because as soon as the difference between the reference and the real position increases a bigger torque is generated due to the particular structure of the controller. The input saturation is present by imposing a ramp instead of the true step reference. In case of a controller based on feedback linearization, this is different. The input $I$ already saturates at the beginning of the ramp in 
TABLE VI

QUALITATIVE CONTROLLER COMPARISON

\begin{tabular}{|l|l|l|l|l|l|l|}
\hline & \multicolumn{3}{|c|}{ Decoupled SISO structure } & Global structure \\
\hline & \multicolumn{4}{|c|}{$\begin{array}{l}\text { Amplitude }=\text { fixed } \\
\text { Phase = controlled }\end{array}$} & \multicolumn{4}{|c|}{ Phase = fixed } \\
\cline { 2 - 7 } & Direct coil & PI-linearized & Flatness & LQR & PQ \\
\hline RMS error & ++++ & ++++ & ++++ & ++ & ++ \\
\hline $\mathrm{e}_{\max }$ & +++ & ++++ & ++++ & + & +++ \\
\hline Power dissipation & - & ++++ & ++++ & ++++ & ++++ \\
\hline Step response & ++++ & + & - & --- & -- \\
\hline Robustness & ++++ & -- & -- & --- & --- \\
\hline
\end{tabular}

order to follow it. When the tracking error starts to increase there is no more room to generate more torque, since the input is already saturated (Fig. 11).

\section{CONCLUSIONS}

This paper presents a variety of control approaches for a dual-stage mechatronic system. It shows that the well-known Park transformation to control PM stepper motors in voltage control can be modified for the use in current control.

Since the system needs to be robust and highly reliable, the only reasonable choice for the final implementation is the decoupled control structure with the direct-coil coarse-stage controller. All the other methods show promising results, especially in terms of energy consumption, but they lack global robustness. In Table VI, a representative comparison of the different control methods is given.

To overcome the lack of torque towards the extremities, another feedback linearization needs to be introduced, which allows action on the amplitude of the input currents $i_{a}$ and $i_{b}$ and the phase of the generated magnetic field. All the new methods would not be applicable anymore, since they are designed for a coarse-stage model transformed to a SISO system.

To determine whether the effects of the blade guiding system and the lead screw cause the robustness issues, one could separate the PM stepper motor from the rest of the system. Such an experience would show whether the controllers work for the PM stepper motor only.

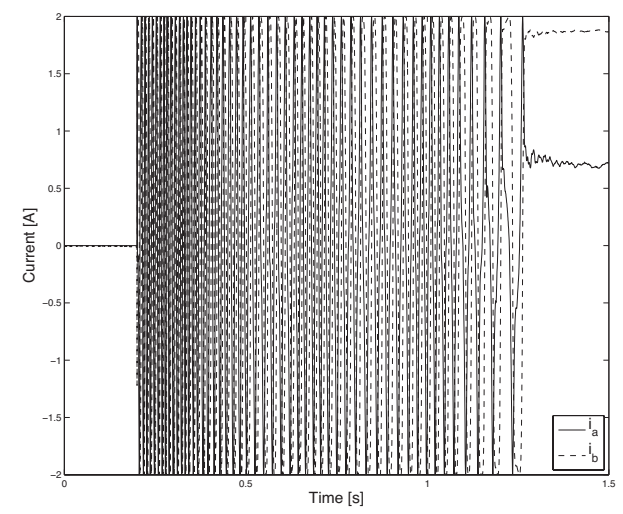

Fig. 11. Inputs $i_{a}$ and $i_{b}$ of the flatness-based controller during a $5000 \mu$ step displacement towards the extremity of the stroke

\section{REFERENCES}

[1] M. Bodson and J. Chiasson, "Application of nonlinear control methods to the positioning of a permanent magnet stepper motor," Proceedings of the 28th IEEE Conference on Desicion and Control, vol. 1, pp. 531-532, December 1989.

[2] M. Bodson, J. N. Chiasson, R. T. Novotnak, and R. B. Rekowski, "High-performance nonlinear feedback control of a permanent magnet stepper motor," IEEE Transactions on Control Systems Technology, vol. 1 , no. 1 , pp. 5-14, 1993.

[3] D. Buccieri, "Jet-scheduling control for flat systems," Ph.D. dissertation, Ecole Polytechnique Fédérale de Lausanne, 2008.

[4] F. Delplancke, S. Lévêque, P. Kervella, A. Glindemann, and L. d'Arcio, "Phase-referenced imaging and micro-arcsecond astrometry with the VLTI," Proc SPIE, vol. 4006, 2000.

[5] F. Delplancke, F. D. F. Paresce, A. Glindemann, F. Lévy, S. Lévêque, and S. Ménardi, "PRIMA for the VLTI - SCIENCE," Astrophysics and Space Science, vol. 286, pp. 99-104, 2003.

[6] J. Deutscher, "A linear differential operator approach to flatness based tracking for linear and non-linear systems," International Journal of Control, vol. 76, no. 3, pp. 266-276, 2003.

[7] A. Glindemann et al., "The VLT Interferometer: a unique instrument for high-resolution astrometry," Proc SPIE, vol. 4006, 2000.

[8] V. Hagenmeyer, Robust nonlinear tracking control based on differential flatness, ser. Mess-, Steuerungs- und Regelungstechnik. VDI Verlag, 2003, no. 978.

[9] S. Henein, "Conception des structures articulées à guidages flexibles de haute précision," Ph.D. dissertation, Ecole Polytechnique Fédérale de Lausanne, 2000. [Online]. Available: http://library.epfl.ch/theses/?nr=2194

[10] X. Hu, W. Guo, T. Huan, and B. Chen, "Discrete-time LQG/LTR dual-stage controller design and implementation for high track density hdds," American Control Conference 1999. Proceedings of the 1999, vol. 6, pp. 4111-41 115, 1999.

[11] R. Launhardt, S. Frink, D. Segransan, and J. Setiawan, "Astrometric Survey for Extra-Solar Planets with PRIMA - Scientific Proposal," Geneva Observatory, Tech. Rep. Planets-PRI-SCI-2001, 2003.

[12] W. C. Messner and H. R. Rapley, "Designing controllers for two stage disk drive actuator systems using the PQ method and the sbode plot," IEEE Transaction on magnetics, vol. 37, no. 2, March 2001.

[13] Y. Michellod, P. Mullhaupt, and D. Gillet, "Strategy for the Control of a Dual-stage Nano-positioning System with a Single Metrology," in 2006 IEEE International Conference on Robotics, Automation and Mechatronics (RAM), December 2006, pp. 1-8.

[14] S. J. Schroeck, W. C. Messner, and R. J. McNab, "On compensator design for linear time-invariant dual-input single-output systems," IEEE/ASME Transactions on Mechatronics, vol. 6, no. 1, pp. 50-57, March 2001.

[15] S.-M. Suh, C. C. Chung, and S.-H. Lee, "Discrete-time LQG/LTR dual-stage controller design in magnetic disk drives," Magnetics, IEEE Transactions on, vol. 37, no. 4, pp. 1891-1895, July 2001.

[16] F. Weibel, Y. Michellod, P. Mullhaupt, and D. Gillet, "Real-time compensation of hysteresis in piezoelectric-stack actuator tracking a stochastic reference," in American Control Conference, 2008.

[17] M. Zribi and J. Chiasson, "Position control of a PM stepper motor by exact linearization," IEEE Transactions on Automatic Control, vol. 36, no. 5, pp. 620-625, May 1991. 